\title{
Evaluation of treatment response to autologous transplantation of noncultured melanocyte/keratinocyte cell suspension in patients with stable vitiligo*
}

\author{
Mariana Gontijo Ramos ${ }^{1,3}$ \\ Camila Gontijo Ramos ${ }^{3}$
}

\author{
Daniel Gontijo Ramos ${ }^{2,3}$
}

DOI: http:/ / dx.doi.org/10.1590/abd1806-4841.20175700

\begin{abstract}
BACKGROUND: Vitiligo is a chronic disease characterized by the appearance of achromic macules caused by melanocyte destruction. Surgical treatments with melanocyte transplantation can be used for stable vitiligo cases.

OвJестіVEs: To evaluate treatment response to the autologous transplantation of noncultured epidermal cell suspension in patients with stable vitiligo.

METHODs: Case series study in patients with stable vitiligo submitted to noncultured epidermal cell suspension transplantation and evaluated at least once, between 3 and 6 months after the procedure, to observe repigmentation and possible adverse effects. The maximum follow-up period for some patients was 24 months.

RESUlTs: Of the 20 patients who underwent 24 procedures, 25\% showed an excellent rate of repigmentation, 50\% good repigmentation, $15 \%$ regular, and $10 \%$ poor response. The best results were observed in face and neck lesions, while the worst in extremity lesions ( $88 \%$ and $33 \%$ of satisfactory responses, respectively). Patients with segmental vitiligo had a better response $(84 \%)$ compared to non-segmental ones $(63 \%)$. As side effects were observed hyperpigmentation of the treated area and the appearance of Koebner phenomenon in the donor area.

STUDY LIMITATIONS: Some limitations of the study included the small number of patients, a subjective evaluation, and the lack of long-term follow-up on the results.

CONCLUSION: Noncultured epidermal cell suspension transplantation is efficient and well tolerated for stable vitiligo treatment, especially for segmental vitiligo on the face and neck.
\end{abstract}

Keywords: Melanocytes; Transplantation, autologous; Treatment outcome; Vitiligo

\section{INTRODUCTION}

Vitiligo is a chronic acquired and progressive disease, characterized by the appearance of achromic macules that are isolated or found in multiple body segments, due to the absence of melanin caused by the destruction or functional loss of melanocytes. These lesions can appear on any part of the body, such as the face and neck, hands, feet, legs, genitals, and hairs. ${ }^{1}$

The world prevalence of vitiligo has been estimated at between $0.5 \%$ and $2 \%$, and can affect individuals from both genders and all types of skin. ${ }^{2}$ The disease can affect individuals of different ages, but it generally begins at around 22 years of age in the USA, 25 in England, and 24 in Brazil. ${ }^{1,3}$ Vitiligo entails a considerable psychological impact as well as an impact on the quality of life of the affected individuals, which can result in relationship problems, low self-esteem, high levels of anxiety, and even depression. ${ }^{4,5}$ According to the review released by the Vitiligo Global Issues Consensus Conference between 2011 and 2012, vitiligo can be classified in three forms: non-segmental vitiligo (NSV), a group which includes the acrofacial, mucosa, generalized, universal and mixed forms; segmental vitiligo (SV), which can be unisegmental, bisegmental, or multisegmental; and the unclassified or undetermined form. ${ }^{6,7}$

The clinical evolution of vitiligo is unpredictable, and the pathogenic mechanisms involved have yet to be fully understood. Nearly one-third of the patients with vitiligo have a family history of the disease, and genetic studies have demonstrated an association with other auto-immune diseases, such as thyroid disease, diabetes, pernicious anemia, Addison's disease, rheumatoid arthritis, among others. ${ }^{2,8}$ The association between vitiligo and the haplotypes of human leukocyte antigens (HLA) also appears to be important in the pathogenesis of the disease in the populations of Brazil and the world. ${ }^{9}$ Among the diverse and complex pathogenic mechanisms involved in the development and clinical evolution of vitiligo are biochemical alterations, neural mechanisms, and intrinsic defects in the adherence and survival of melanocytes. ${ }^{7}$ The auto-immune hypothesis, in which cells and molecules of the immunological sys-

\footnotetext{
Received on 15.02.2016.

Approved by the Advisory Board and accepted for publication on 16.05.2016.

Work conducted in a private clinic in Belo Horizonte (MG), Brazil.

Financial Support: Fapemig.

Conflict of Interest: None.

College of Human, Social, and Health Sciences, Universidade Fumec - Belo Horizonte (MG), Brazil

Dermatology Clinic, Santa Casa de Belo Horizonte - Belo Horizonte (MG), Brazil.

Private clinic - Belo Horizonte (MG), Brazil.

C2017 by Anais Brasileiros de Dermatologia
} 
tem act together and end up provoking the death of melanocytes, seems to play an important role in the pathogenesis of vitiligo and is currently the most well-accepted among specialists. ${ }^{1,7}$ The immunological activity also seems to be involved in the capacity of response to the different types of therapeutic approaches to treat vitiligo. ${ }^{10-12}$

There are many surgical and non-surgical therapeutic approaches used to treat vitiligo, but none can guarantee the complete and long-lasting disappearance of the disease. The main approaches include treatments with corticosteroids as well as topical and orally administered immunomodulators; phototherapy with sunlight, UVA, PUVA, UVB radiation, especially of narrow band (NB-UVB), and excimer lasers. Some antioxidants and other natural products, such as Polypodium leucotomos, can also be used, mainly in association with other treatments. ${ }^{2,13}$

Surgical treatments may be recommended as the treatment of choice for cases of segmental vitiligo, as well as for patients with a stable disease and who did not respond well to the other types of treatment. Surgery can also be recommended for areas of difficult treatment, including the hands, feet, and mucosa. Skin grafts and epidermal cell suspensions are the most commonly applied approaches. ${ }^{14}$

The transplantation of epidermal cells in suspension can be performed with cultivated cells, but this would require a longer period of time, more laboratory resources, and high costs. Moreover, it is difficult to guarantee the feasibility of the culture. By contrast, larger areas can be treated. In the non-cultured cell transplants, these can be obtained by scraping, curettage, suction blister, or by removal of a thin layer of skin with a Blair blade or other resection techniques..$^{15}$ The transplantation of epidermal cell suspension (melanocytes and keratinocytes) has been used and documented by different groups, primarily in Asia, the Middle East, and Europe, as well as in some groups in Brazil. ${ }^{13-18}$

The aim of the present study is to evaluate the patient response with stable vitiligo in different areas of the body for treatment with the transplantation of noncultured epidermal cell suspension.

\section{METHODS}

Patients with stable vitiligo were selected to undergo treatment. The stability was evaluated by the medical history reported by the patient, indicating the non-appearance of new lesions or modifications in the already existing lesions over a period of one year. This study included 20 patients, male and female, between 10 and 50 years of age, and lesions found on different parts of the body, treated during 2013. Patients with more than $30 \%$ of the body surface involved, pregnant women, and patients with skin cancer were excluded from the study. The patients were not undergoing any other types of treatment for vitiligo when they received treatment through the transplant of melanocytes. During the clinical evaluation, the patients were classified as regards the phototype and type of vitiligo.

The technique employed was that described by Mulekar (2004), with minimal changes, which was performed in one or two sessions in each patient. ${ }^{19}$ Photographs were taken of the treated areas at the time of transplant and during follow-up after the procedure.

The donor area used in this study was the upper and anterior region of the thigh, in a proportion of up to 1:10 in relation to the area to be treated. In the donor area, antisepsis with chlorhexidine and anesthesia with local infiltration of $2 \%$ lidocaine $($ Xylestesin $=$ $2.0 \%$ with vasoconstrictor $=2 \%$ lidocaine hydrochloride + epinephrine hemitartrate (1:200,000 in epinephrine). The skin was stretched and a thin layer (approximately $200 \mu \mathrm{m}$ ) was removed by shaving with a flexible blade (Personna). A Cicaplast Baume B5 (La Roche Posay) cream was applied on the surface of the wound, which was covered with a gauze and micropore surgical tape (3M).

The thin layer of skin was transferred to a Petri plate, with the epidermis turned right side up, containing approximately $4 \mathrm{~mL}$ of $0.2 \%$ trypsin solution and EDTA, and was incubated for approximately 20 minutes in an incubator at $37^{\circ} \mathrm{C}$. The trypsin solution was then discarded, and the skin sample was washed in DMEM F12 medium (Cultilab, São Paulo). The dermis was separated from the epidermis using delicate anatomical tweezers and fragmented into small pieces. The solution with skin fragments in DMEM F12 medium were transferred to a $15 \mathrm{~mL}$ tube and centrifuged at $1200 \mathrm{rpm}$ for 6 minutes to obtain a cell pellet. The pellet was re-suspended in a DMEM F12 medium in a $1 \mathrm{~mL}$ syringe. The volume was chosen according to the size of the area to be treated, varying between 0.1 and $0.5 \mathrm{~mL}$.

The receptor area received the application of a topical anesthesia (4\% HCL Tetracaine, 4\% HCL Lidocaine, 4\% HCL Prilocaine in a cream vehicle, Equilibrium pharmacy) 30 minutes before the beginning of the procedure. The receptor area was submitted to dermabrasion with an electric dermabrasion device (Beltec, LB 100, São Paulo) until the papillary dermis (bleeding dew point). Next, the area was covered with a gauze, damp with saline solution, until the moment of cell transplant. The prepared cell suspension was spread uniformly with a $1 \mathrm{~mL}$ syringe through the entire receptor area after the gauze was removed and was covered again with a collagen dressing (Neuskin-F, Medira, UK). The dressing was then finalized, using sterilized gauze, transparent adhesive dressing (Tegaderm), and fixed with micropore surgical tape. The patient rested for 20 minutes and was then released with recommendations to rest for one week, with as little movement of the treated area as possible and not to practice activities that could cause perspiration or that would wet the treated areas. The patients returned to the doctor's office after seven days and the dressings were removed. It was recommended for the patient to expose the treated area to sunlight for 10 minutes daily.

The patients were evaluated between 3 and 12 months after the procedure. The change in pigmentation was evaluated, by a dermatologist on the patient and in comparison with photographs taken before and after the procedure. The repigmentation rates were determined through a previously described scoring system: excellent ( $\geq 90 \%$ repigmentation), good (50 to $89 \%$ repigmentation), regular (20 to $49 \%$ repigmentation), and poor (<20\% repigmentation). The rate considered satisfactory was greater than $50 \%$ of repigmentation (sum of the results considered excellent and good), such as that used by researchers. ${ }^{20}$ Adverse effects during the procedure, such as pain and discomfort, were observed and registered and, after the procedure, the coloration and aspect of the treated areas were evaluated as compared to the adjacent skin. The occurrence of hyperpigmentation, diffuse hypopigmentation or hypopigmentation on the edges was evaluated. The aspect of the donor area was also 
evaluated. The study was sent to the Brazil platform and approved by an ethics committee, logged under report number 1,346,262.

\section{RESULTS}

During the period of the study, 20 patients were submitted to a total of 24 procedures of autologous transplantation of epidermal cell suspension (melanocytes and keratinocytes) to treat vitiligo in different body areas. The results were evaluated between 3 and 6 months after the interventions. Some patients were followed up for up to 24 months after the procedure. The lesions were treated in two sessions in four patients: in two patients (12 and 14), to complement the partial repigmentation obtained in the first session, with results considered satisfactory after two sessions $(90 \%$ and $70 \%$, respectively). Patient 5, with acrofacial vitiligo, was submitted to two sessions in different areas and presented excellent results in the facial area (forehead, 100\%), but a poor result in the region of the fingertips (20\%). By contrast, patient 13 , with segmental vitiligo in the internal area of the thigh, obtained a poor response in the first session, but did show a good response in the second attempt in the same area (80\%). The characteristics of each patient and the response to the treatment are represented in table 1.

The majority of patients treated were women - 14 patients (70\%) - while six were men (30\%). The average age was of 30.8 years, the majority $(55 \%)$ presented phototype III and segmental vitiligo (60\%). The characteristics of the study's sample are listed in table 2.

Results of excellent repigmentation were observed in $25 \%$ of the patients. Ten patients (50\%) presented a good rate of repigmentation, $15 \%$ presented regular repigmentation, and 10\% presented a poor response (Figure 1). Of the total of treated patients, $75 \%$ obtained a satisfactory result ( $>50 \%$ repigmentation).

The repigmentation rates, according to the location of the lesions, are presented in table 3 . The best responses were observed in the face and neck regions, with $37.5 \%$ presenting an excellent re- sponse ( $\geq 90 \%$ repigmentation) and $50 \%$ presenting a good result (50 to $89 \%$ repigmentation). For the lesions identified on the limbs, the results were also considered satisfactory $(20 \%$ excellent and

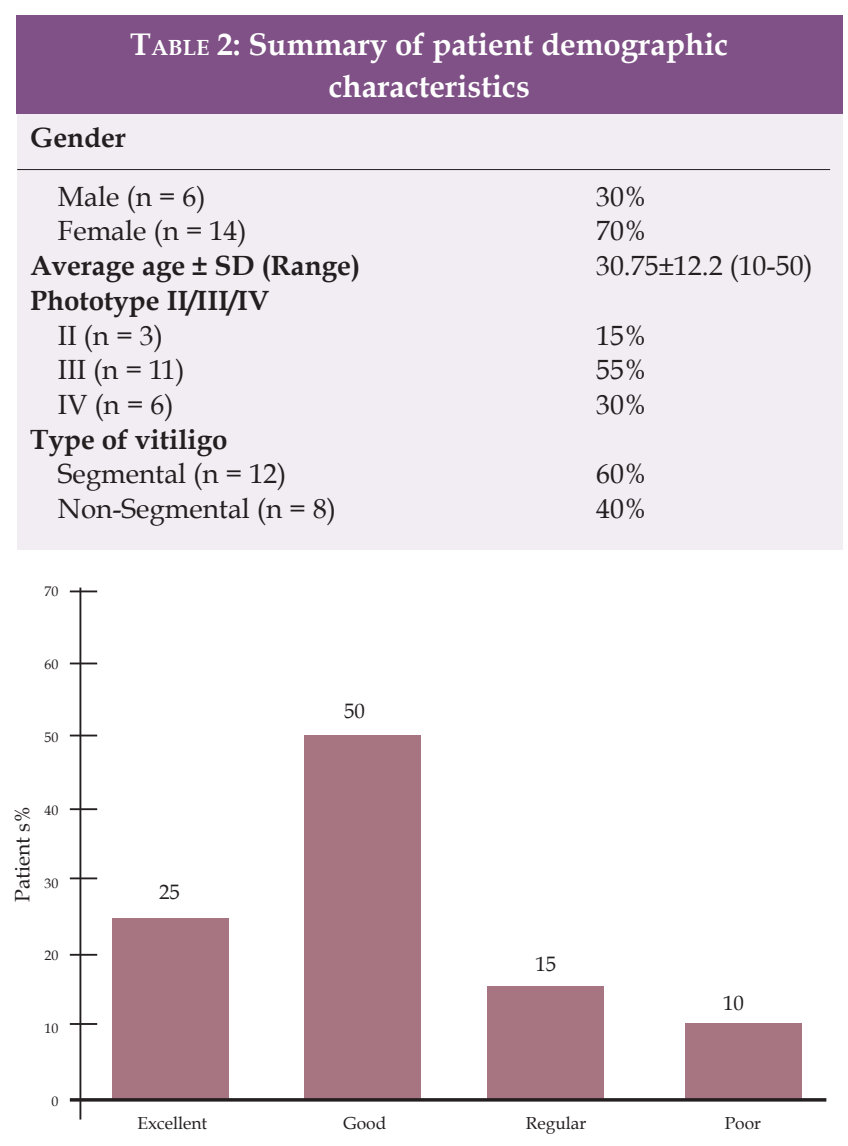

FIgURE 1 : Evaluation of repigmentation for all cases. Excellent $=\geq$ $90 \%$, Good $=50$ to $89 \%$, Regular $=20$ to $49 \%$, and Poor $=<20 \%$

\section{TABLE 1: Characteristics of the patients and repimentation rates observed after treatment}

\begin{tabular}{lllllll} 
Patient & Gender & Age & Phototype & Type of vitiligo & Treated areas/sessions & Repigmentation (\%) \\
\hline 1 & F & 41 & III & Segmental & Face/1 & 80 \\
2 & F & 27 & II & Generalized & Areola, armpits, and wrists/1 & 0 \\
3 & F & 29 & III & Segmental & Trunk/1 & 90 \\
4 & F & 24 & II & Segmental & Trunk/1 & 20 \\
5 & M & 35 & IV & Acrofacial & Face and hands/2 & $100 / 20-60$ \\
6 & F & 14 & IV & Generalized & Feet/1 & 80 \\
7 & F & 25 & III & Segmental & Face/1 & 70 \\
8 & M & 49 & III & Segmental & Face/1 & 100 \\
9 & F & 10 & IV & Segmental & Neck/1 & 80 \\
10 & F & 19 & IV & Generalized & Arms/1 & 80 \\
11 & F & 31 & III & Generalized & Face and knees/1 & 40 \\
12 & F & 31 & III & Generalized & Knees/2 & 90 \\
13 & F & 28 & IV & Segmental & leg/2 & 80 \\
14 & F & 48 & III & Generalized & Knees/2 & 70 \\
15 & M & 26 & III & Segmental & Genitals/1 & 0 \\
16 & F & 17 & III & Segmental & Neck/1 & 80 \\
17 & F & 50 & III & Segmental & Trunk/1 & 80 \\
18 & M & 20 & II & Segmental & Face/1 & 90 \\
19 & M & 44 & III & Generalized & Armpits/1 & 40 \\
20 & M & 47 & IV & Segmental & Trunk/1 & 90
\end{tabular}




\begin{tabular}{lllll}
\hline \multicolumn{4}{c}{ TABLE 3: Evaluation of the repigmentation rates by } \\
location of lesions \\
Response/Area & Face/neck & Limbs & Trunk & Acral \\
\hline Excellent & $37,5 \%$ & $20 \%$ & $25 \%$ & 0 \\
Good & $50 \%$ & $60 \%$ & $12,5 \%$ & $33.3 \%$ \\
Regular & 0 & $20 \%$ & $25 \%$ & $33.3 \%$ \\
Poor & $12,5 \%$ & 0 & $37,5 \%$ & $33.3 \%$ \\
Satisfactory Rate* & $88 \%$ & $80 \%$ & $38 \%$ & $33 \%$
\end{tabular}

Excellent $=\geq 90 \%$, Good $=50$ to $89 \%$, Regular $=20$ to $49 \%$, and Poor $=<20 \%$

* Rate considered satisfactory $=>50 \%$

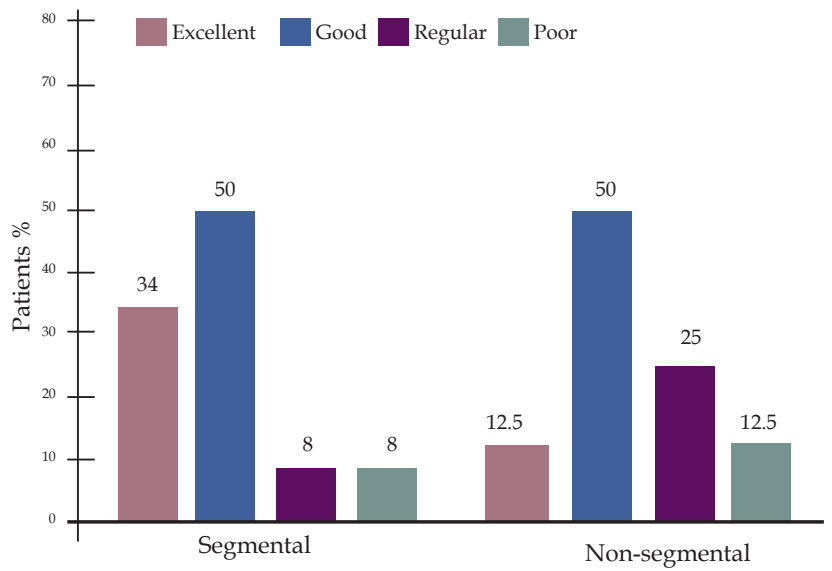

FIGURE 2: Evaluation of repigmentation in patients with segmental vitiligo $(n=12)$ and non-segmental vitiligo $(n=8)$. Excellent $=\geq 90 \%$, Good $=50$ to $89 \%$, Regular $=20$ to $49 \%$, and Poor $=<20 \%$
$60 \%$ good). The lesions on the trunk presented $37.5 \%$ satisfactory responses, while the worst rates of repigmentation were observed for the extremities (acral), in which there was no excellent response and $33 \%$ presented satisfactory results.

As regards the type of vitiligo, $34 \%$ of patients (four) who presented segmental vitiligo obtained an excellent repigmentation rate. Six patients (50\%) presented a good response, $8 \%$ regular, and $8 \%$ poor. For the non-segmental vitiligo, the rates were of $12.5 \%$ (one patient) for excellent responses, 50\% (four patients) good, $25 \%$ regular, and $12.5 \%$ of poor responses (Figure 2). Figures 3 and 4 show the repigmentation of the lesions of patients with segmental and non-segmental vitiligo, respectively.

The main adverse event associated with the procedure was pain due to the anesthetic infiltration in the donor area, and especially in the dermabrasion area (previously prepared with topical anesthetic cream, occluded for 30 minutes), in addition to burning during the placing of the collagen dressing. In general, the procedure was well tolerated and the majority of the patients appeared to be willing to undergo the procedure again, if necessary. The pain after the procedure occurred mainly in the most sensitive areas, and none of the patients developed infections in donor or receptor areas. One case of Koebner phenomenon appeared in the donor region (thigh) (Figure 5). Hyperchromia in the receptor area in two patients and slight hypopigmentation in another patient also were observed. The uniform coloration was obtained in the majority of the receptor areas of other patients.


FIGURE 3 : Patient with segmental vitiligo on the face. Before treatment (A), six months (B), and two years (C) after the treatment with noncultured epidermal cell suspension transplantation
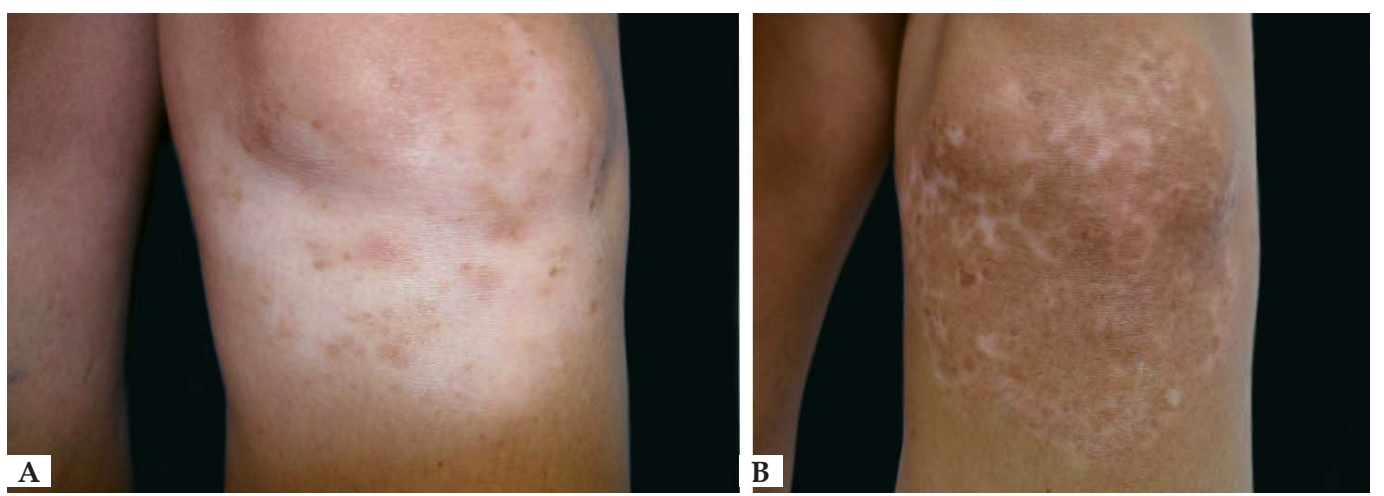

Figure 4: Patient with non-segmental vitiligo on the knee. Before treatment (A) and six months (B) after treatment with non-cultured epidermal cell suspension transplantation 




FigURE 5 : Koebner phenomenon in the donor region (thigh) after treatment with noncultured epidermal cell suspension transplantation

\section{DISCUSSION}

Surgery is one of the therapeutic options to treat stable segmental vitiligo and other clinical forms that do not respond or respond poorly to other treatments. This alternative has been widely used by dermatologists from different countries. ${ }^{15}$ There are different techniques of surgical treatment, with thin partial skin grafts, punches or micropunches, and suction blistering representing the most common techniques. Cell transplantation represents a distinct type of treatment, as it consists of the acquisition of epidermal cells concentrated in the fluid medium. This procedure can be performed with previously cultured cells or with noncultured cells, as was used in the present study. According to Mulekar (2016), this method represents a middle ground between skin grafts, which can have a slightly better success rate but that treats only smaller area lesions, and cultured cell transplantation, which treats larger areas but has a high cost and requires the use of laboratories that are specialized in cell cultures. ${ }^{21}$ Noncultured melanocyte/keratinocyte suspension transplantation (also called epidermal cells) is a procedure that requires the use of some laboratory equipment and techniques but that can be executed within a short period of time (around $2 \mathrm{~h}$ ), in outpatient doctor's offices or dermatological clinics. This method allows the treatment of a wide range of body regions and larger areas, with a ratio of up to 1:10 of the donor area. For better results, the recommended treatment is a ratio of $1: 5$, that is, to treat an area that is five times greater than the donor area. ${ }^{21,22}$

The technique used in the present study was that defined by Olsson \& Juhlin in 1998 and later modified by Mulekar in 2004. ${ }^{19,23}$ In scientific literature, it is possible to find different modifications in the methodology of this procedure, from the manner in which to obtain the cells from the donor area, to the preparation of the receptor area, to the types of dressings used. Epidermal cells can be obtained by resection of a thin layer of skin, using a flexible blade, through the epidermal curettage or through manual dermabrasion. ${ }^{18,21,24}$ The receptor area can be prepared by manual or motorized dermabrasion device, $\mathrm{CO}_{2}$ lasers, liquid nitrogen, among others. ${ }^{25}$ One recent article published by Al-Hadidi et al. (2016) suggests that post-operative care is also essential to the success of the treatment. The dressings placed in the receptor area, such as the collagen dressings used in our study, guarantee the adherence and survival of the transplanted cells, thus stimulating the healing process. The authors also highlighted that minimizing the possibilities of bacterial contamination and mechanical, chemical, and phototoxic traumas is also important in order to increase the viability of the melanocytes and allow an efficient repigmentation. ${ }^{25}$

In the present study, the repigmentation rates were evaluated in 20 patients treated with autologous transplantation of epidermal cells (melanocytes/keratinocytes). It was possible to observe that, of the total number of patients, the majority presented a satisfactory response (75\%) after treatment, with $25 \%$ presenting an excellent response and $50 \%$ presenting a good response. A similar result was reported by El-Zawahr et al. (2011), when treating 23 cases of stable vitiligo with the same technique, who observed an excellent response in $23 \%$ of the patients. ${ }^{26}$ Huggins et al. (2012) observed a lower satisfactory response, of only $48 \%$, with $17 \%$ presenting excellent results and $31 \%$ presenting good results. ${ }^{22}$ One recent study conducted by Komen et al. (2015), using the ReCell ${ }^{\mathrm{TM}}$ device to obtain cell suspension, reported a satisfactory response in $60 \%$ of the patients, which was also slightly lower than that observed in the present study. ${ }^{27} \mathrm{~A}$ greater percentage of satisfactory responses was observed by Bao et al. (2015) when treating patients with segmental and non-segmental vitiligo with melanocyte transplantation. ${ }^{20}$ The authors observed $53 \%$ of the results with excellent repigmentation and $28 \%$ with good repigmentation, with noncultured cell suspension. In their study, the authors also compared three different surgical techniques, treating, in the same session, the lesion area divided into three parts (suction blistering grafting, cultured epidermal cell suspension transplantation, and noncultured cell transplantation). They concluded that the three methods are safe and efficient and should be chosen according to the size and location of the lesions and available laboratory resources. ${ }^{20}$

Although there are some differences in the responses observed by different groups that used similar transplant methods, there is a consensus that some factors, such as the type of vitiligo, can determine the greater or lesser success of the treatment. Mulekar et al. suggest that in patients with segmental vitiligo it is possible to achieve a nearly $95 \%$ repigmentation by applying noncultured melanocyte/keratinocyte transplantation, with a low recurrence of lesions in the treated patients. ${ }^{28}$ However, when these same authors treated patients with non-segmental vitiligo, the success rates were reduced to $67 \% .{ }^{29}$ In the present study, this tendency was also observed, with better rates of satisfactory repigmentation in patients with segmental vitiligo ( $34 \%$ excellent and $50 \%$ good).

The location of the lesions is a determining factor in the success of different modalities of treatment for vitiligo. In this study, the best repigmentation rates were observed in the lesions located in the face and cervical regions, followed by the limbs (arms and legs). The worst results were observed in the trunk and extremities (hands and feet). Similar results were demonstrated by Huggins et al. (2012). ${ }^{22}$ Other authors have also reported excellent responses in the facial region. ${ }^{28,29}$ The presence of vitiligo in the extremities, especially on the fingertips, is associated with a worse response to different types of treatment for vitiligo. ${ }^{30-32}$ Mulekar (2016) reports that, even if a 
small number of patients with acral lesions can have satisfactory responses, the majority present a quick relapse. ${ }^{21}$ As regards the trunk, the results are varied, according to different groups. Some observed worse responses when compared to those observed in the present study, while others demonstrated more favorable responses. ${ }^{20,22}$ Many of these differences still cannot be fully explained due to the complex mechanisms related to the pathogenesis of the vitiligo and to diverse factors that can influence the course and response to different treatments.

The main adverse effects noted in the patients treated with epidermal cell suspension in the present study included pain and discomfort during the stages of the procedure and in the immediate post-operative stage. Greater complications were not observed, such as infections and scars in the donor/receptor areas. The majority of patients reported satisfaction with the methods and availability to undergo the procedure again, if necessary. This study identified one patient with the appearance of Koebner phenomenon in the donor area, while another patient presented hyperpigmentation with depigmented edges in the receptor area. These effects have also been observed in other studies. ${ }^{22,28,32}$ The possibility of the occurrence of these effects should be explained to the patients before beginning the procedure.

Vitiligo is a multifactorial disease of complex pathogenesis that is not fully understood, as it is difficult to explain the difficulty of repigmentation in some locations and even in cases which would apparently have a good chance of response, but that present no significant improvement.

One important factor in the selection of patients for surgical treatment with epidermal cell suspension is the stability of the disease. Clinical stability is determined by the non-appearance of new lesions and by the absence of changes in the existing lesions. Rao et al. (2012) suggest stability of a year to achieve about 70\% success, but the study's sample size was small. ${ }^{33}$ In our clinical practice, what is considered for surgical procedures are the patients with clinical stability of at least one year, a parameter considered appropriate by other researchers as well. ${ }^{21,34}$

Though clinical stability is important in the selection of patients, it is believed that the auto-immune mechanisms involved in the development and progression of vitiligo are much more complex and difficult to understand. Studies have demonstrated that clinical stability does not always exclude the presence of an auto-immune process in patients with vitiligo. Rao et al. (2012) demonstrated an increase in the quantity of $\mathrm{T} C \mathrm{CD} 8+$ and CD45RO+ lymphocytes in the vitiligo lesion areas, and this increase was related to the lower stability and worse response to treatment with melanocyte transplants. ${ }^{33}$ The presence of cytotoxic lymphocytes in the skin lesions has also been demonstrated by other studies. ${ }^{35,36}$ In addition to the immunological mechanisms that act in the site, the systemic immunological state of the patient can also influence the pathogenesis and the results of the treatment for vitiligo. The presence of anti-melanocyte antibodies; the increase in pro-inflammatory cytokines, such as the tumor necrosis factor and interferon gamma; and the changes in regulatory $\mathrm{T}$ cells also seem to play an important role. ${ }^{7,37,38}$ Other factors, such as molecular characteristics of suspension cells and neurogenic and biochemical factors, still require better comprehension. ${ }^{21,36}$ Nevertheless, the evaluation of these molecular parameters is difficult and uncommon in the routine of a clinical practice, occurring mainly in some scientific research.

The present study does present some limitations. A relatively small sample of patients was used. The evaluation of repigmentation was determined by subjective analysis; however, this method is frequently used in studies on vitiligo. Other evaluation methods that could be used include visual scale instruments, such as the Vitiligo Area Scoring Index (VASI), and devices for the digital analysis of images.

It is also important to emphasize that an interesting alternative is the combined treatment, which is already in use in clinical routines and that can also be applied to epidermal cell suspension transplants. In addition to sunlight, recommended for patients after transplants, the treatment with excimer laser and NB-UVB, together with the melanocyte transplant, appear to significantly increase repigmentation rates. ${ }^{39,40}$

\section{CONCLUSIONS}

The epidermal cell, melanocyte/keratinocyte, transplantation procedure is one of the most appropriate methods for the treatment of stable vitiligo, presenting good responses primarily in lesions located in the face and limbs. Both patients and dermatologists should have realistic expectations regarding the evolution and treatment of the disease and choose the therapeutic options according to the appropriate criteria. All in all, many questions still remain unanswered and others will still arise regarding vitiligo, its pathogenesis, and its treatment. $\square$ 


\section{REFERENCES}

1. Tarlé RG, Nascimento LM, Mira MT, Castro CC. Vitiligo--part 1. An Bras Dermatol. 2014;89:461-70.

2. Whitton ME, Pinart M, Batchelor J, Leonardi-Bee J, González U, Jiyad Z, et al. Interventions for vitiligo. Cochrane Database Syst Rev. 2015:CD003263.

3. de Barros JC, Machado Filho CD, Abreu LC, de Barros JA, Paschoal FM, Nomura MT, et al. A study of clinical profiles of vitiligo in different ages: an analysis of 669 outpatients. Int J Dermatol. 2014;53:842-8.

4. Boza JC, Kundu RV, Fabbrin A, Horn R, Giongo N, Cestari TF. Translation, crosscultural adaptation and validation of the vitiligo-specific health-related quality of life instrument (VitiQoL) into Brazilian Portuguese. An Bras Dermatol. 2015;90:358-62.

5. Nogueira LSC, Zancanaro PCQ, Azambuja RD. Vitiligo e emoções. An Bras Dermatol. 2009;84:41-41-5.

6. Faria AR, Tarlé RG, Dellatorre G, Mira MT, Castro CC. Vitiligo--Part 2- classification, histopathology and treatment. An Bras Dermatol. 2014;89:784-90.

7. Mohammed GF, Gomaa AH, Al-Dhubaibi MS. Highlights in pathogenesis of vitiligo. World J Clin Cases. 2015;3:221-30.

8. Nunes DH, Esser LM. Epidemiological profile of vitiligo patients and its association with thyroid disease. An Bras Dermatol. 2011;86:241-8.

9. Ramire LD, Marcos EV, Godoy DA, de Souza-Santana FC. Association of class and II HLA alleles and haplotypes with susceptibility to vitiligo: a study of patients withvitiligo from southeast Brazil. Int J Dermatol. 2016;55:e347-55

10. Ingordo V, Gentile C, Iannazzone SS, Cusano F, Naldi L. Vitiligo and autoimmunity: an epidemiological study in a representative sample of young Italian males. J Eur Acad Dermatol Venereol. 2011;25:105-9.

11. Steitz J, Brück J, Lenz J, Büchs S, Tüting T. Peripheral CD8+ T cell tolerance against melanocytic self-antigens in the skin is regulated in two steps by CD4+ T cells and local inflammation: implications for the pathophysiology of vitiligo. J Invest Dermatol. 2005;124:144-50.

12. Oyarbide-Valencia K, van den Boorn JG, Denman CJ, Li M, Carlson JM, Hernandez $\mathrm{C}$, et al. Therapeutic implications of autoimmune vitiligo T cells. Autoimmun Rev. 2006;5:486-92.

13. Daniel BS, Wittal R. Vitiligo treatment update. Australas J Dermatol. 2015;56:85-92.

14. Whitton M, Pinart M, Batchelor JM, Leonardi-Bee J, Gonzalez U, Jiyad Z, et al. Evidence-Based Management of vitiligo: summary of a Cochrane systematic review. Br J Dermatol. 2016;174:962-9.

15. Mulekar SV, Isedeh P. Surgical interventions for vitiligo: an evidence-based review. Br J Dermatol. 2013;169:57-66.

16. Ebrahimi A, Radmanesh M, Kavoussi H. Recipient site preparation for epidermal graft in stable vitiligo by a special fraise. An Bras Dermatol. 2015;90:55-60.

17. Ramos MG, Ramos DG, Gontijo G, Ramos CG, Rocha TN, Rocha RH. Noncultured melanocyte/keratinocyte transplantation for the treatment of stable vitiligo on the face: report of two cases. An Bras Dermatol. 2013;88:811-3.

18. Machado Filho CD, Timoner FR. Epidermal curettage technique (ECT) for tissue harvest from the donor area for melanocyte autologous grafting in cases of vitiligo. An Bras Dermatol. 2014;89:681-3.

19. Mulekar SV. Long-term follow-up study of segmental and focal vitiligo treated by autologous, noncultured melanocyte-keratinocyte cell transplantation. Arch Dermatol. 2004;140:1211-5

20. Bao H, Hong W, Fu L, Wei X, Qian G, Xu A. Blister roof grafting, cultured melanocytes transplantation and non-cultured epidermal cell suspension transplantation in treating stable vitiligo. A mutual self-control study. J Dermatolog Treat. 2015;26:571-4.

21. Mulekar SV. Melanocyte-keratinocyte transplantation procedure: A few insights. Indian J Dermatol Venereol Leprol. 2016;82:13-5.

22. Huggins RH, Henderson MD, Mulekar SV, Ozog DM, Kerr HA, Jabobsen G, et al. Melanocyte-keratinocyte transplantation procedure in the treatment of vitiligo: the experience of an academic medical center in the United States. J Am Acad Dermatol. 2012;66:785-93.

23. Olsson MJ, Juhlin L. Leucoderma treated by transplantation of a basal cell layer enriched suspension. Br J Dermatol. 1998;138:644-8.

24. Gupta S, Kumar A, Mahendra A, Gupta S. A minimally invasive, scarless technique of donor tissue harvesting for noncultured epidermal cell suspensiontransplantation in vitiligo. J Am Acad Dermatol. 2015;73:e213-5.

25. Al-Hadidi N, Griffith JL, Al-Jamal MS, Hamzavi I.. Role of recipiente-site preparation techniques and post operative wound dressings in the surgical managementof vitiligo. J Cutan Aesthet Surg. 2015;8:79-87.

26. El-Zawahry BM, Zaki NS, Bassiouny DA, Sobhi RM, Zaghloul A, Khorshied MM, et al. Autologous melanocyte-keratinocyte suspension in the treatment of vitiligo. J Eur Acad Dermatol Venereol. 2011;25:215-20.
27. Komen L, Vrijman C, Tjin EP, Krebbers G, de Rie MA, Luiten RM, et al. Autologous cell suspension transplantation using a cell extraction device in segmental vitiligo and piebaldism patients. A randomized controlled pilot study. J Am Acad Dermatol. 2015;73:170-2.

28. Isedeh P, Al Issa A, Lim HW, Mulekar SS, Mulekar SV. Uncommon responses of segemental vitiligo to melanocyte-keratinocyte transplantation procedure. J Cutan Med Surg. 2015;19:177-81.

29. Mulekar, SV. Long-term follow-up study of 142 patients with vitiligo vulgaris treated by autologous, non-cultured melanocyte-keratinocyte cell transplantation. Int J Dermatol. 2005;44:841-5.

30. Guerra L, Capurro S, Melchi F, Primavera G, Bondanza S, Cancedda R, et al. Treatment of "stable" vitiligo by Timed surgery and transplantation of cultured epidermal autografts. Arch Dermatol. 2000;136:1380-9.

31. Holla AP, Sahni K, Kumar R, Parsad D, Kanwar A, Mehta SD. Acral vitiligo and lesions over joints treated with non-cultured epidermal cell suspension transplantation. Clin Exp Dermatol. 2013;38:332-7.

32. Paul M. Autologous non-cultures basal-cell enriched epidermal cell suspension transplantation in vitiligo: India Experience. J Cutan Aesthet Surg. 2011;4:23-8.

33. Rao A, Gupta S, Dinda AK, Sharma A, Sharma VK, Kumar G, et al. Study of clinical,biochemical and immunological factors determining stability of disease in patients withgeneralized vitiligo undergoing melanocyte transplantation. $\mathrm{Br} \mathrm{J}$ Dermatol. 2012;166:1230-6.

34. Parsad D, Gupta S; IADVL Dermatosurgery Task Force. Standard guidelines of care for vitiligo surgery. Indian J Dermatol Venereol Leprol. 2008;74:S37-45.

35. Zhou MN1, Zhang ZQ, Wu JL, Lin FQ, Fu LF, Wang SQ, et al. Dermal mesenchymal stem cells (DMSCs) inhibit skin-homing CD8 + T cell activity, a determining factor of vitiligo patient's autologous melanocytes transplantation efficiency. PLoS One. 2013;8:e60254.

36. Speeckaert R, Speeckaert MM, van Geel N. Why treatments do(n't) work in vitiligo: An autoinflammatory perspective. Autoimmun Rev. 2015;14:332-40.

37. Zhu MC, Liu CG, Wang DX, Zhan Z. Detection of serum anti-melanocyte antibodies and and identification of related antigens in patients with vitiligo. Genet Mol Res. 2015;14:16060-73.

38. Hegab DS, Attia MA. Decreased circulatins T regulatory cells in egyptian patients with nonsegmental vitiligo: correlation with disease activity. Dermatol Res Pract. 2015;2015:145409.

39. Ebadi A, Rad MM, Nazari S, Fesharaki RJ, Ghalamkarpour F, Younespour S. The additive effect of excimer laser on noncultured melanocyte-keratinocyte transplantation for the treatment of vitiligo: a clinical trial in an Iranian population. J Eur Acad Dermatol Venereol. 2015 Apr;29:745-51.

40. Zhang DM, Hong WS, Fu LF, Wei XD, Xu AE. A randomized controlled study of the effects of different modalities of narrow-band ultraviolet B therapy on the outcome of cultured autologous melanocytes transplantation in treating vitiligo. Dermatol Surg. 2014;40:420-6.

MAILING ADDRESS:

Mariana Gontijo Ramos

Av. do Contorno, 4747 - Sala 709 - Serra

30110-001 - Belo Horizonte - MG

Brazil

E-mail:ramosbh@yahoo.com.br

How to cite this article: Ramos MG, Ramos DG, Ramos CG. Evaluation of treatment response to autologous transplantation of noncultured melanocyte/keratinocyte cell suspension in patients with stable vitiligo. . An Bras Dermatol. 2017;92(3):312-8. 